\title{
Positive Tensor Network Approach for Simulating Open Quantum Many-Body Systems
}

\author{
A. H. Werner, ${ }^{1}$ D. Jaschke, ${ }^{2,3}$ P. Silvi, ${ }^{2}$ M. Kliesch, ${ }^{1}$ T. Calarco, ${ }^{2}$ J. Eisert, ${ }^{1}$ and S. Montangero ${ }^{2}$ \\ ${ }^{1}$ Dahlem Center for Complex Quantum Systems, Freie Universität Berlin, 14195 Berlin, Germany \\ ${ }^{2}$ Institute for Complex Quantum Systems \& Center for Integrated Quantum Science and Technologies (IQST), \\ Universität Ulm, 89069 Ulm, Germany \\ ${ }^{3}$ Department of Physics, Colorado School of Mines, Golden, Colorado 80401, USA
}

(Received 5 February 2015; revised manuscript received 18 February 2016; published 7 June 2016)

Open quantum many-body systems play an important role in quantum optics and condensed matter physics, and capture phenomena like transport, the interplay between Hamiltonian and incoherent dynamics, and topological order generated by dissipation. We introduce a versatile and practical method to numerically simulate one-dimensional open quantum many-body dynamics using tensor networks. It is based on representing mixed quantum states in a locally purified form, which guarantees that positivity is preserved at all times. Moreover, the approximation error is controlled with respect to the trace norm. Hence, this scheme overcomes various obstacles of the known numerical open-system evolution schemes. To exemplify the functioning of the approach, we study both stationary states and transient dissipative behavior, for various open quantum systems ranging from few to many bodies.

DOI: 10.1103/PhysRevLett.116.237201

Open quantum systems are ubiquitous in physics. To some extent any quantum system is coupled to an environment, and in many instances this interaction significantly alters the system's dynamics. Traditionally, such decoherence processes are seen as adversary to coherent state manipulation. However, suitably engineered dissipation can also have beneficial effects and can be exploited for state preparation [1-8], even of states containing strong entanglement or featuring topological order. In condensed matter physics, many concepts such as transport are often studied within the closed systems paradigm, but it is becoming increasingly clear that some familiar concepts may have to be revisited in the open system setting [9], where the interplay between Hamiltonian interactions and dissipation leads to interesting physical effects.

Since few analytical methods are available for such systems, the design of novel numerical tools for the simulation of dissipative quantum many-body systems is of the utmost importance. In this work, we present a new algorithm that captures open many-body dynamics in one spatial dimension - for both transient and steady regimesbased on a locally purified tensor network Ansatz class. It comprises a new approach in that the positivity of the operators is maintained during the whole simulation. Importantly, the approximation errors can be controlled in a way that yields a trace-norm certificate. Hence, the algorithm provides not only a conceptually new approach to the problem, but also combines several desired features of existing schemes and overcomes previous limitations.

Tensor-network Ansatz classes have proven to be successful in capturing the physics of many-body states [10-15] by parametrizing a very small but physically relevant submanifold of quantum states with local correlations. The density-matrix renormalization method [16] can indeed be viewed as a variational principle over matrixproduct states [11,13,17-19]. Generalizing these ideas, a number of exciting methods have been proposed [20-26], some of which also allow us to study open quantum systems. In most cases matrix-product operators (MPOs) are at the heart of these methods. Indeed, several variants have already been developed [27-35], many of which exploit the well-known features of tensor network Ansätze to encode the mixed many-body quantum states in a compact matrix-product formulation, ultimately making the algorithm efficient and stable both for transient [27-29] and steady state physics [34].

However, in such a MPO description, the resulting truncated operators may not be positive; in fact, this property cannot even be tested locally, because it comprises a computationally intractable problem [36]. In Ref. [27] this is circumvented by dropping the positivity assumption during the time evolution, which requires that the approximation errors remain sufficiently small. Alternatively, quantum jump schemes make use of a stochastic unraveling of the master equation $[30,37,38]$ and then employ purestate techniques, at the expense of having to sample over many realizations. The comparative performance of these two approaches has been recently investigated [39].

Remarkably, the subset of matrix-product operators that are cast in a locally purified tensor network (LPTN) form [27,40] shows promising features: such operators are positive by construction and exhibit all the helpful features typical of tensor networks. However, while the LPTN structure has been studied to represent boundary states in projected entangled pair tensor networks $[26,41,42]$ a practical algorithm for one-dimensional open systems has yet to be formulated. Here, we show that such a positivitypreserving algorithm can actually be engineered for 

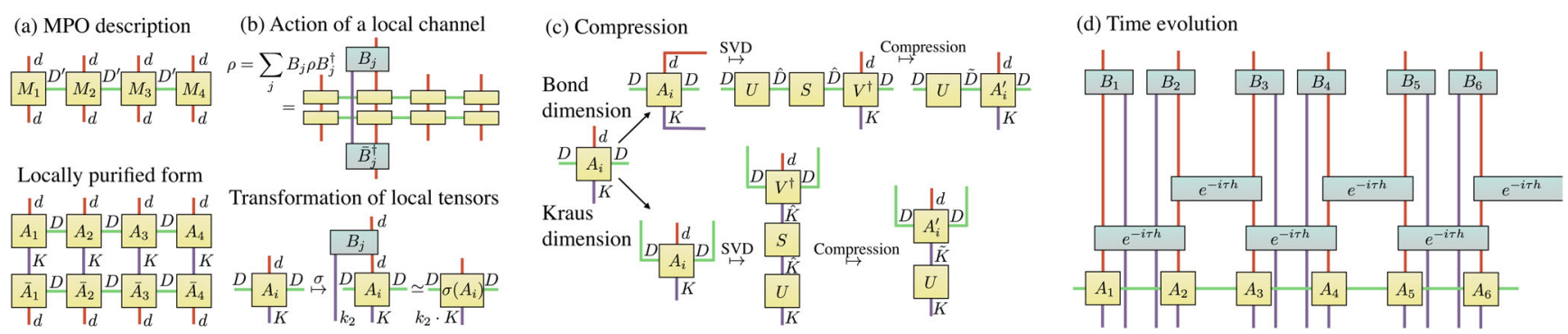

FIG. 1. Markov dynamics of a quantum spin chain on the level of local tensors. Panel (a) shows the relationship between a density matrix $\rho$ in the MPO representation (top) and the locally purified tensor network (bottom) with tensors $A_{l}$, physical dimension $d$, bond dimension $D^{\prime}$, and Kraus dimension $K$. (b) Action of a local channel $T$ acting exclusively on lattice site 2 on the level of the MPO and on the level of the locally purified tensors. In the latter, the Kraus rank $k_{2}$ of the quantum channel $T$ is joined together with $K$. (c) Compression schemes for the bond and Kraus dimension of a local tensor via singular value decompositions (SVD). (d) Locally purified evolution of a time step $e^{\tau \mathcal{L}}$ for a nearest neighbor Hamiltonian and on-site Lindblad operators. We only show three of the five Trotter-Suzuki layers from Eq. (3).

Markovian dynamics: this scheme has the computational efficiency of tensor network methods, allows us to control all approximation errors in the operationally relevant trace norm, and preserves positivity by construction, thus ultimately merging the advantages of previous techniques while solving known issues.

Algorithm.-Our goal is to simulate the evolution of spin chains under local Markovian dynamics, i.e., onedimensional lattice systems (at finite system size, with open boundary conditions) governed by the Lindblad master equation

$$
\frac{d \rho}{d t}=\mathcal{L}(\rho)=-i[H, \rho]+\mathcal{D}(\rho) .
$$

Here, $H=\sum_{j} H_{j}$ is the Hamiltonian and the dissipative part of the Lindblad generator $\mathcal{L}$ takes the form $\mathcal{D}(\rho)=\sum_{\alpha}\left(L_{\alpha} \rho L_{\alpha}^{\dagger}-\left\{L_{\alpha}^{\dagger} L_{\alpha}, \rho\right\} / 2\right)$, where the Lindblad operators $L_{\alpha}$ model the coupling of the system with the environment. We focus on the typical scenario, where the elementary Hamiltonian terms $H_{j}$ as well as the Lindblad operators $L_{\alpha}$ are two local, meaning that they only couple spins on neighboring sites, and denote them by $H^{[l, l+1]}$ or $L^{[l, l+1]}$, respectively.

We describe the variational mixed state of the system as a tensor network representing the density matrix $\rho$. But instead of expressing $\rho$ directly as a MPO $[27,43]$ we keep it at every stage of our algorithm in its locally purified form $\rho=X X^{\dagger}$, where the purification operator $X$ is decomposed as a variational tensor network

$$
[X]_{r_{1}, \ldots, r_{N}}^{s_{1}, \ldots, s_{N}}=\sum_{m_{1}, \ldots, m_{N-1}} A_{m_{1}}^{[1] s_{1}, r_{1}} A_{m_{1}, m_{2}}^{[2] s_{2}, r_{2}} \cdots A_{m_{N-1}}^{[N] s_{N}, r_{N}}
$$

with $1 \leq s_{l} \leq d, 1 \leq r_{l} \leq K$, and $1 \leq m_{l} \leq D$. Hence, we represent $\rho$ by a locally purified tensor network consisting of rank-4 tensors $A^{[l]}$ with physical dimension $d$, bond dimension $D$, and Kraus dimension $K$ [see Fig. 1(a)].
Our algorithm is an extension of the time evolving block decimation (TEBD) scheme [44], acting on the level of the local tensor $A^{[l]}$ that also allows for dissipative channels, and never requires us to contract the two tensor network layers $\left(X\right.$ and $\left.X^{\dagger}\right)$ together. Similarly to TEBD, we split the propagator $e^{\tau \mathcal{L}}$ for a small time step $\tau$ into several dissipative Trotter-Suzuki layers [45] of mutually commuting operators. Let us consider the evolution from time $t$ to $t+\tau$ in row-wise vectorization $\left.\left.\left|\rho_{t+\tau}\right\rangle\right\rangle=|e\rangle\right\rangle^{\tau \mathcal{L}} \rho_{t}=$ $\left.e^{\tau(-i H \otimes 1+i \mathbb{} \otimes \bar{H}+\mathcal{D})}\left|\rho_{t}\right\rangle\right\rangle$, where $\left.|M\rangle\right\rangle$ denotes the vector given as the row-wise concatenation of a matrix $M$. As usual, for one spatial dimension (for possible generalizations to higher dimensions see the Supplemental Material [46]) we define the operators $H_{o}$ and $H_{e}$ by splitting the Hamiltonian $H=\sum_{l=1}^{N} H^{[l, l+1]}$ into two sums, one containing the even interactions $H^{[2 l, 2 l+1]}$ and one containing the odd interactions $H^{[2 l-1,2 l]}$. If the Lindblad operators $L_{\alpha}$ act only on site (the case of two-site Lindblad operators is treated later on), we can approximate $e^{\tau \mathcal{L}}$ using a second order Trotter-Suzuki formula as (see the Supplemental Material [46])

$$
e^{\tau \mathcal{L}}=e^{\tau \mathcal{H}_{o} / 2} e^{\tau \mathcal{H}_{e} / 2} e^{\tau \mathcal{D}} e^{\tau \mathcal{H}_{e} / 2} e^{\tau \mathcal{H}_{o} / 2}+O\left(\tau^{3}\right),
$$

partially shown in Fig. $1(\mathrm{~d})$, where $\mathcal{H}_{\nu}=-i H_{\nu} \otimes \mathbb{1}+i \mathbb{1} \otimes \bar{H}_{\nu}$ with $\nu=o, e$. The layers $\mathcal{H}_{e}$ and $\mathcal{H}_{o}$ implement the coherent part of the evolution and are identical to the usual TEBD layers. Expressing $\rho_{t}$ as $\rho_{t}=X_{t} X_{t}^{\dagger}$ we find that by setting $X_{t}^{\prime}=e^{-i \tau \mathcal{H}_{\nu} / 2} X_{t}$ we recover exactly $\rho_{t}{ }^{\prime}=e^{\tau \mathcal{H}_{\nu} / 2} \rho_{t}$. Hence, for the coherent part of the dynamics, we can just adapt the usual TEBD algorithm for nearest neighbor Hamiltonians.

The dissipative layer requires a more careful treatment and we exploit the fact that since the operators $L^{[l]}$ act only on a single site, we find $e^{\tau \mathcal{D}}=\underset{l}{\otimes} e^{\tau \mathcal{D}^{[l]}}$, with $\mathcal{D}^{[l]}=$ $\sum_{j}\left[L_{j}^{[l]} \otimes \bar{L}_{j}^{[l]}-\left(L_{j}^{[l] \dagger} L_{j}^{[l]} \otimes \mathbb{1}+\mathbb{1} \otimes L_{j}^{[l] T} \bar{L}_{j}^{[l]}\right)\right] / 2$, where 
the sum runs over all Lindblad operators $L_{j}^{[l]}$ acting on site $l$. Since $e^{\tau \mathcal{D}^{[l]}}$ is completely positive, we can find a set of Kraus operators $\left\{B_{l, q}\right\}$ satisfying $e^{\tau \mathcal{D}^{[l]}}=$ $\sum_{q=1}^{k} B_{l, q} \otimes \bar{B}_{l, q}$. The action of $e^{\tau \mathcal{D}^{[l]}}$ on the level of the local tensors is now given by a contraction of $B_{l, q}$ into $A_{t}^{[l]}$, while joining the variational Kraus dimension $K$ with the Kraus rank $k \leq d^{2}$ of the quantum channel $e^{\tau \mathcal{D}^{[l]}}$, as shown in Fig. 1(b). The application of each Trotter-Suzuki layer increases only the dimension of a single index of the local tensors $A^{[l]}$ : the bond dimension $D$ is increased by the coherent layers, and the Kraus dimension $K$ by the dissipative layers. This allows for immediate compression of the enlarged dimension similar to the standard density matrix renormalisation group setting. In all compression steps the Frobenius norm error introduced on the purification operators can be kept track of. This translates into a trace-norm error for the state itself. By taking also the error from the Trotter-Suzuki approximation into account, we obtain an explicit bound for the trace-norm error; see Theorem 1 in the Supplemental Material [46].

Numerical results. - In order to verify the applicability of our method we consider three prototypical benchmark situations. The first one comprises a few-body scenario, consisting of two qubits coupled via cavities with additional excitation losses [52]. As a genuine many body example we study the steady state of an $X X Z$ spin- $1 / 2$ chain with edge dissipation channels, which allows for comparison with analytical solutions derived in Ref. [9]. Finally, we show the validity of the two-site Lindbladoperator approach in the case of the Kitaev wire [7].

In the first model, two interacting optical cavities $\left(C_{1}\right.$ and $C_{2}$ ) are each coupled to a private qubit $\left(S_{1}\right.$ and $\left.S_{2}\right)$. Ordering the sites as $\left(S_{1}-C_{1}\right)-\left(C_{2}-S_{2}\right)$ gives a nearest neighbor model suited for our algorithm. The coherent part of the dynamics is captured by the Jaynes-Cummings Hamiltonian, which describes each spin-cavity interaction, plus a photon tunneling between cavities. In terms of the spin operators $\sigma_{l}^{ \pm}=\left(\sigma_{l}^{x} \pm i \sigma_{l}^{y}\right) / 2$ and the creation $c_{l}^{\dagger}$ and annihilation operators $c_{l}$ of the cavity photons, the Hamiltonian is given by $H=\sum_{l=1,2}\left(\alpha_{l}\left(\sigma_{l}^{+} c_{l}+\sigma_{l}^{-} c_{l}^{\dagger}\right)+\right.$ $\left.\omega_{C} n_{l}+\omega_{S} \sigma_{l}^{z}\right)+\alpha_{C C}\left(c_{1}^{\dagger} c_{2}+c_{2}^{\dagger} c_{1}\right)$. The dissipation models a homogeneous probability of excitation losses and is given by single-site Lindblad operators: $L_{S_{l}}=\sqrt{\gamma} \sigma_{l}^{-}$for the spins and $L_{C_{l}}=\sqrt{\gamma} c_{l}$ for the cavities. We start the evolution in a pure product state, where only the right cavity is nonempty and filled exactly with $N(0)=3$ photons. The symmetries of the model imply an easy relation between the total excitation number $\mathcal{N}(t)$ and the coupling strength $\gamma: \mathcal{N}(t)=\mathcal{N}(0) \mathrm{e}^{-\gamma t}$, a behavior that is reproduced by our simulations with high precision (under $0.2 \%$ relative deviation). Figure 2 shows the occupation on each site as well as $\mathcal{N}(t)$, which correctly reproduces the expected exponential decay. The inset shows the infidelity

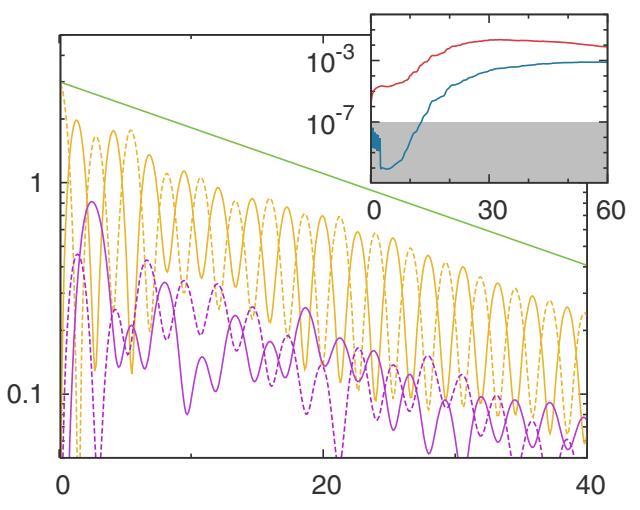

FIG. 2. Main: excitation populations of the four sites (see the main text) in the coupled spin-cavity model (yellow, cavity; violet, spin; dashed, right; straight, left), here for $\gamma=0.05$, $\alpha_{1}=\alpha_{2}=0.48, \alpha_{C C}=-1.0, \omega_{C}=\omega_{S}=1.0$, as well as their sum $\mathcal{N}$ (green line). The latter is nicely fitted by an exponential, with decay rate $\gamma_{\text {fit }}=0.04997 \pm 8 \times 10^{-5}$. Inset: comparison of the locally purified evolution, here for bond dimension $D=40$ and Kraus dimension $K=40$, with the exact Liouville evolution: infidelity $\mathcal{I}$ (blue line) and relative Hilbert-Schmidt distance (red line). Infidelities are estimated to be numerically reliable above $10^{-7}$ (nonshaded area).

$\mathcal{I}\left(\rho_{1}, \rho_{2}\right)=1-\operatorname{Tr}\left[\left(\rho_{2}{ }^{1 / 2} \rho_{1} \rho_{2}{ }^{1 / 2}\right)^{1 / 2}\right]$ of the locally purified evolution $\rho_{1}$ with respect to the exact evolution $\rho_{2}$ carried out in Liouville space. As expected, close to the steady state the deviation of the locally purified dynamics from the exact evolution converges to a finite value (depending both on $\tau$ and the maximal bond $D$ and Kraus dimension $K$ ).

As a second benchmark, we consider the evolution of a spin-chain under the $X X Z$ Hamiltonian $H=$ $\sum_{l}\left(\sigma_{l}^{x} \sigma_{l+1}^{x}+\sigma_{l}^{y} \sigma_{l+1}^{y}+\Delta \sigma_{l}^{z} \sigma_{l+1}^{z}\right)$. Via a Jordan-Wigner transformation this system is mapped into a spinless fermion Hubbard model with a density-density nearest neighbor interaction. Therefore, with the addition of two reservoirs embodied by Markov channels at the edges, it models fermionic dc transport in a quantum wire. We introduce the Lindblad operators $L_{\leftarrow}=\sqrt{2 \gamma} \sigma_{1}^{+}$at the leftmost site (source) and $L_{\rightarrow}=\sqrt{2 \gamma} \sigma_{N}^{-}$at the rightmost site (drain). We identify the steady state for different parameter regimes $(\Delta, \gamma)$. For comparison with analytic results from Ref. [9], we consider the local $z$ axis magnetization $\sigma_{l}^{z}$ and the spin-current operator $I_{l}=$ $i\left(\sigma_{l}^{+} \sigma_{l+1}^{-}-\sigma_{l}^{-} \sigma_{l+1}^{+}\right)$. The steady state regime is achieved by evolving the system until these observables become stationary. Figure 3 shows the local magnetization of a chain of $N=100$ spins in the top panel, while the current as a function of the chain length is plotted on the bottom frame. A remarkable quantitative match to Ref. [9] emerges even for small $D$ and $K(\sim 60)$.

Finally, we consider a setting with two-local instead of on-site Lindblad operators (see the Supplemental Material [46] for details). We employ a two-layer Trotter-Suzuki 


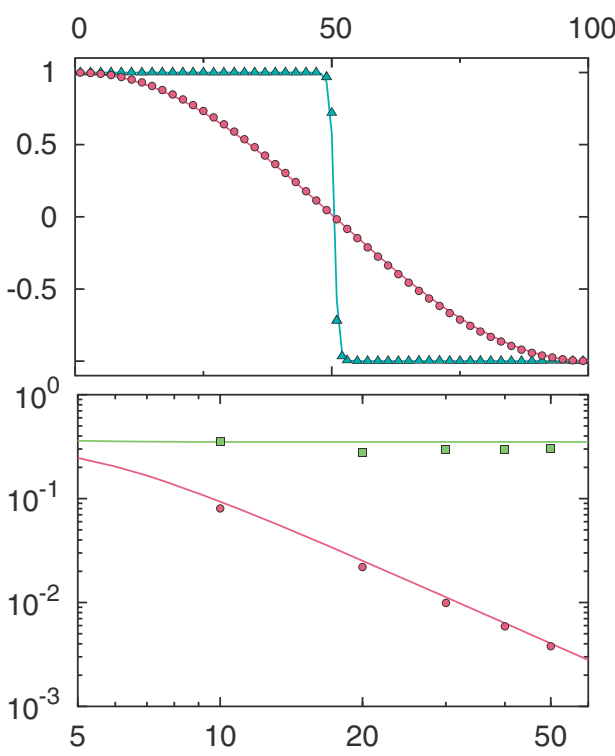

FIG. 3. Comparison of simulated steady state (points) with analytical results (lines) from Ref. [9] for the $X X Z$ model with edge driving of $\gamma=1$ in several parameter regimes. Green squares, $\Delta=0.5$; red dots, $\Delta=1.0$; cyan triangles, $\Delta=1.5$. Top: local magnetization in the $z$ direction $\left\langle\sigma_{j}^{z}\right\rangle$ as a function of the site $j$, for a chain of length $N=100$. Bottom: time averaged steady state spin current $I_{j}=2 \operatorname{Im}\left\langle\sigma_{j}^{+} \sigma_{j+1}^{-}\right\rangle$at the chain center $j=N / 2$, as a function of the total chain length $N$.

approximation with odd $\mathcal{L}_{o}=\mathcal{H}_{o}+\mathcal{D}_{o}$ and even $\mathcal{L}_{e}=$ $\mathcal{H}_{e}+\mathcal{D}_{e}$ terms. After computing the Kraus decomposition for the corresponding nearest-neighbor channels $e^{\tau \mathcal{L}_{2 l, 2 l+1}}=$ $\sum_{q}^{k} B_{q}^{[2 l, 2 l+1]} \otimes \bar{B}_{q}^{[2 l, 2 l+1]}$ one can choose how to implement the action of $B_{q}^{[2 l, 2 l+1]}$ onto $A^{[2 l]}$ and $A^{[2 l+1]}$. In particular, there are different possibilities for distributing the Kraus rank $k$ of the channel between the Kraus dimensions $K_{2 l}$ and $K_{2 l+1}$ of the two sites.

Moreover, when such a dimension $k$ is distributed nontrivially $\left(k_{1}>1\right.$ to the left site, and $k_{2}>1$ to the right site, where $k_{1} k_{2}=k$ ) there is an additional freedom, represented by a unitary transformation $U$ in the $k$-dimensional auxiliary space, that influences the precision of the algorithm. This gauge transformation $U$ is discussed in detail in the Supplemental Material [46], alongside a numerical technique we adopt to optimize it. For an appropriate comparison, we consider three strategies: (a) Kraus rank all to one side ( e.g., $k_{2}=1$ ), (b) Kraus rank distributed as evenly as possible $\left(k_{1} \simeq k_{2} \simeq \sqrt{k}\right.$ ), with random $U$ (unoptimized), and (c) analogously to (b) but with optimized $U$.

As a benchmark of this technique we simulated a Kitaev wire model, comparing the LPTN to the exact evolution, which we discuss in detail in the Supplemental Material [46]. The results showed that we can capture accurately the realtime evolution starting from an entangled mixed random state, by direct comparison of our scheme with the exact Liouville evolution, for a chain of six sites. It also suggests that strategies (a) and (c) yield, surprisingly, equivalent precision, and are preferable choices to strategy (b).

Discussion and advantages of the scheme.-The LPTN algorithm introduced in this Letter yields an overall computational cost scaling as $O\left(d^{5} D^{3} K\right)+O\left(d^{5} D^{2} K^{2}\right)$, by executing a clever contraction of the coherent terms. Moreover, this method takes advantage of the gauge freedom, e.g., by reducing costs for local measurements from $O(N)$ to $O(1)$, with $N$ being the system size. It complements known evolution schemes employed in many-body calculations for 1D systems (namely the MPO representation and the quantum trajectories technique) in a significant way. Indeed, our scheme allows us to overcome known shortcomings of the other methods, although possibly concomitant with a slightly reduced efficiency, as we describe briefly in the following.

The computational cost of regular MPO techniques scales as $O\left(d^{8} \tilde{D}^{2}\right)+O\left(d^{6} \tilde{D}^{3}\right)$. They have been extensively employed with success [27-33,35]. The LPTN paradigm is a preferable choice in cases where the negativity of the MPO Ansatz becomes pathological. Note, however, that the roles of the bond dimensions in the schemes are not identical [40], and there is an additional trade-off between $D$ and $K$ in the LPTN case. Quantum trajectory techniques, in contrast, carry a computational cost (assuming the single sample evolution relies on a matrix product state Ansatz with bond dimension $\hat{D})$ of the order $O\left(\eta d^{4} \hat{D}^{2}\right)+$ $O\left(\eta d^{3} \hat{D}^{3}\right)$, where $\eta$ is the number of samples employed in the stochastic unraveling. These methods have delivered excellent accuracy in a number of physically relevant scenarios, especially in transient dynamics and in situations where the stationary states are expected to be close to being pure [30]. Their limitations are crucial, however, when the stationary states are expected to be highly mixed, such as in high temperature environments. Then, the number of samples required increases drastically, challenging also a parallel computation approach. Possibly more challenging is the fact it can happen that quantum states of relatively small correlations are represented as ensembles of matrix product states each with a large bond dimension $\hat{D}$. An extreme case of this form is constituted by a maximally mixed stationary state with $D=1$ and $K=d$, represented as an ensemble of pure states of bond dimension $\hat{D}$, giving rise to an overhead of $\hat{D}^{3}$.

Importantly, the LPTN offers a concise control of errors accumulated during the simulation and guarantees a simulation to be accurate up to a given error. At the same time, the new scheme introduced here calls for further theoretical and numerical studies to determine under which physical conditions and dynamical processes each of these three different approaches is most effective. A deeper understanding of these issues will guide future research in out-ofequilibrium quantum many-body systems providing the best possible numerical tool available in each different scenario. 
Perspectives.-In this work, we have introduced a versatile algorithm for simulating open quantum many-body systems. All errors made by the algorithm are bounded in the trace norm. The ideas presented here overcome a number of previous limitations and allow us to probe both transient dynamics and stationary behavior. We have discussed three important benchmark cases, and a number of perspectives open up here. First, the framework can be used to analyze weakly interacting open quantum systems, perturbing frequently studied free fermionic models to study topology generated by engineered dissipation [7,8]. Clearly, notions of algebraic and exponential dissipation can readily be accessed [35], as well as glasslike dynamics [53] and kinematic inhibitance, or the interplay between localization by dissipation and disorder. Furthermore, the method finds immediate application in the dissipative quantum engineering of entangled many-body states [54], for instance, by merging with optimal control techniques [55]. It also allows us to explore shortcuts to adiabaticity [56] in open-system quantum many-body settings. Another intriguing enterprise is to investigate the stability of stationary states under local Liouvillian perturbations, in particular, without the assumption of a finite log-Sobolev constant or rapid mixing $[57,58]$. It would also be exciting to explore formulations of our method in a time-dependent variational principle framework $[14,18]$.

This work has been supported by the EU (SIQS, RAQUEL, COST, AQuS), the ERC (TAQ), the DFG (SFB/TRR21, EI 519/7-1, CRC 183), and the BadenWürttemberg Stiftung (Eliteprogramm for Postdocs). We thank T. Prosen for discussions. We acknowledge the BWgrid for computational resources.

[1] S. Diehl, A. Micheli, A. Kantian, B. Kraus, H. P. Buechler, and P. Zoller, Nat. Phys. 4, 878 (2008).

[2] F. Verstraete, M. M. Wolf, and J. I. Cirac, Nat. Phys. 5, 633 (2009).

[3] H. Krauter, C. A. Muschik, K. Jensen, W. Wasilewski, J. M. Petersen, J. I. Cirac, and E. S. Polzik, Phys. Rev. Lett. 107, 080503 (2011).

[4] M. B. Plenio and S. F. Huelga, Phys. Rev. Lett. 88, 197901 (2002).

[5] J. Eisert and T. Prosen, arXiv:1012.5013.

[6] M. J. Kastoryano, M. M. Wolf, and J. Eisert, Phys. Rev. Lett. 103, 110501 (2009).

[7] S. Diehl, E. Rico, M. A. Baranov, and P. Zoller, Nat. Phys. 7, 971 (2011).

[8] C.-E. Bardyn, M. A. Baranov, C. V. Kraus, E. Rico, A. Imamoglu, P. Zoller, and S. Diehl, New J. Phys. 15, 085001 (2013).

[9] T. Prosen, Phys. Rev. Lett. 107, 137201 (2011).

[10] M. Fannes, B. Nachtergaele, and R. F. Werner, Lett. Math. Phys. 25, 249 (1992).

[11] D. Perez-Garcia, F. Verstraete, M. M. Wolf, and J. I. Cirac, Quantum Inf. Comput. 7, 401 (2007).
[12] V. Murg, F. Verstraete, and J. I. Cirac, Phys. Rev. A 75, 033605 (2007)

[13] U. Schollwöck, Ann. Phys. (Amsterdam) 326, 96 (2011).

[14] C. M. Dawson, J. Eisert, and T. J. Osborne, Phys. Rev. Lett. 100, 130501 (2008).

[15] J. Eisert, M. Cramer, and M. B. Plenio, Rev. Mod. Phys. 82, 277 (2010).

[16] S. R. White, Phys. Rev. Lett. 69, 2863 (1992).

[17] S. Östlund and S. Rommer, Phys. Rev. Lett. 75, 3537 (1995).

[18] J. Haegeman, J. I. Cirac, T. J. Osborne, I. Pizorn, H. Verschelde, and F. Verstraete, Phys. Rev. Lett. 107, 070601 (2011).

[19] R. Orus, Ann. Phys. (Amsterdam) 349, 117 (2014).

[20] M. Rizzi, S. Montangero, and G. Vidal, Phys. Rev. A 77, 052328 (2008).

[21] M. Rizzi, S. Montangero, P. Silvi, V. Giovannetti, and R. Fazio, New J. Phys. 12, 075018 (2010).

[22] M. Gerster, P. Silvi, M. Rizzi, R. Fazio, T. Calarco, and S. Montangero, Phys. Rev. B 90, 125154 (2014).

[23] P. Corboz, G. Evenbly, F. Verstraete, and G. Vidal, Phys. Rev. A 81, 010303(R) (2010).

[24] T. Barthel, C. Pineda, and J. Eisert, Phys. Rev. A 80, 042333 (2009).

[25] T. Barthel, M. Kliesch, and J. Eisert, Phys. Rev. Lett. 105, 010502 (2010).

[26] M. Lubasch, J. I. Cirac, and M.-C. Bañuls, Phys. Rev. B 90, 064425 (2014).

[27] F. Verstraete, J. J. Garcia-Ripoll, and J. I. Cirac, Phys. Rev. Lett. 93, 207204 (2004).

[28] M. Zwolak and G. Vidal, Phys. Rev. Lett. 93, 207205 (2004).

[29] L. Bonnes, D. Charrier, and A. M. Läuchli, Phys. Rev. A 90, 033612 (2014).

[30] A. J. Daley, Adv. Phys. 63, 77 (2014).

[31] C. Karrasch, J. H. Bardarson, and J. E. Moore, Phys. Rev. Lett. 108, 227206 (2012).

[32] C. Karrasch, J. H. Bardarson, and J. E. Moore, New J. Phys. 15, 083031 (2013).

[33] I. Pižorn, V. Eisler, S. Andergassen, and M. Troyer, New J. Phys. 16, 073007 (2014).

[34] J. Cui, J. I. Cirac, and M. C. Bañuls, Phys. Rev. Lett. 114, 220601 (2015).

[35] Z. Cai and T. Barthel, Phys. Rev. Lett. 111, 150403 (2013).

[36] M. Kliesch, D. Gross, and J. Eisert, Phys. Rev. Lett. 113, 160503 (2014).

[37] H. Pichler, J. Schachenmayer, A. J. Daley, and P. Zoller, Phys. Rev. A 87, 033606 (2013).

[38] S. Sarkar, S. Langer, J. Schachenmayer, and A. J. Daley, Phys. Rev. A 90, 023618 (2014).

[39] L. Bonnes and A. M. Laeuchli, arXiv:1411.4831.

[40] G. de las Cuevas, N. Schuch, D. Perez-Garcia, and J. I. Cirac, New J. Phys. 15, 123021 (2013).

[41] I. Pižorn, L. Wang, and F. Verstraete, Phys. Rev. A 83, 052321 (2011).

[42] M. Lubasch, J. I. Cirac, and M.-C. Bañuls, New J. Phys. 16, 033014 (2014).

[43] B. Pirvu, V. Murg, J. I. Cirac, and F. Verstraete, New J. Phys. 12, 025012 (2010).

[44] G. Vidal, Phys. Rev. Lett. 91, 147902 (2003). 
[45] M. Kliesch, T. Barthel, C. Gogolin, M. Kastoryano, and J. Eisert, Phys. Rev. Lett. 107, 120501 (2011).

[46] See Supplemental Material http://link.aps.org/supplemental/ 10.1103/PhysRevLett.116.237201 for an in-depth analysis of our algorithm including discussions about convergence, error bounds, and extensions to more general physical scenarios. It contains Refs. [47-51].

[47] J. Watrous, arXiv:1207.5726.

[48] M. Suzuki, J. Math. Phys. (N.Y.) 32, 400 (1991).

[49] U. Schollwoeck, Ann. Phys. (Amsterdam) 326, 96 (2011).

[50] M.-D. Choi, Linear Algebra Appl. 10, 285 (1975).

[51] N. Schuch, M. M. Wolf, F. Verstraete, and J. I. Cirac, Phys. Rev. Lett. 98, 140506 (2007).

[52] S. Schmidt, D. Gerace, A. A. Houck, G. Blatter, and H. E. Türeci, Phys. Rev. B 82, 100507 (2010).
[53] D. Poletti, P. Barmettler, A. Georges, and C. Kollath, Phys. Rev. Lett. 111, 195301 (2013).

[54] Y. Lin, J. P. Gaebler, F. Reiter, T. R. Tan, R. Bowler, A. S. Sorensen, D. Leibfried, and D. J. Wineland, Nature (London) 504, 415 (2013).

[55] P. Doria, T. Calarco, and S. Montangero, Phys. Rev. Lett. 106, 190501 (2011).

[56] G. Vacanti, R. Fazio, S. Montangero, G. M. Palma, M. Paternostro, and V. Vedral, New J. Phys. 16, 053017 (2014).

[57] M. J. Kastoryano and J. Eisert, J. Math. Phys. (N.Y.) 54, 102201 (2013).

[58] T. S. Cubitt, A. Lucia, S. Michalakis, and D. Perez-Garcia, Commun. Math. Phys. 337, 1275 (2015). 